\title{
Judging familiarity and emotion from very brief musical excerpts
}

\author{
SuZANNe FilipiC \\ Université de Bourgogne, CNRS-UMR 5022, Dijon, France \\ BARBARA TILlMANN \\ Université de Claude Bernard Lyon, CNRS-UMR 5020, Lyon, France \\ AND \\ EMmanuel Bigand \\ Institut Universitaire de France \\ and Université de Bourgogne, CNRS-UMR 5022, Dijon, France
}

\begin{abstract}
In the present study, the gating paradigm was used to measure how much perceptual information that was extracted from musical excerpts needs to be heard to provide judgments of familiarity and of emotionality. Nonmusicians heard segments of increasing duration $(250,500,1,000 \mathrm{msec}$, etc.). The stimuli were segments from familiar and unfamiliar musical excerpts in Experiment 1 and from very moving and emotionally neutral musical excerpts in Experiment 2. Participants judged how familiar (Experiment 1) or how moving (Experiment 2) the excerpt was to them. Results show that a feeling of familiarity can be triggered by $500-\mathrm{msec}$ segments, and that the distinction between moving and neutral can be made for $250-\mathrm{msec}$ segments. This finding extends the observation of fast-acting cognitive and emotional processes from face and voice perception to music perception.
\end{abstract}

Everyday experience suggests that getting a sense of how familiar or moving a musical piece is can be very fast. When turning on the radio, we can rapidly select the music we know best and/or enjoy most. The time course of processes involved in triggering a feeling of familiarity or identifying a stimulus or an emotion has been investigated with faces, language, and sounds, but rarely with music. For the other stimuli, previous studies have reported fast-acting processes: Spoken words can be recognized after $240 \mathrm{msec}$ (Grosjean, 1980), famous voices can be identified after $250 \mathrm{msec}$ (Schweinberger, Herholz, \& Sommer, 1997), and $160 \mathrm{msec}$ of a vocal sound are enough to distinguish between excitement and calmness (Rapoport, 1997). Fast-acting processes are also reflected in early event-related potentials (ERPs), for example, for the processing of facial familiarity and expression (170 and $120 \mathrm{msec}$, respectively; Ashley, Vuilleumier, \& Swick, 2004; Eimer \& Holmes, 2007) and of emotions in voices (200 msec; Wambacq, Shea-Miller, \& Abubakr, 2004).

For music, it has been shown that cognitive processes can be triggered by chords after 500 msec (Tekman \& Bharucha, 1998) and $170 \mathrm{msec}$ (Bigand, Poulin, Tillmann, $\&$ D'Adamo, 2003) and that unexpected chords evoke early ERPs (200 msec; Koelsch, Gunter, Friederici, \& Schröger, 2000). For fast-acting cognitive processes, a striking finding was that 100 -msec excerpts from original recordings of popular songs was sufficient for their identification (Schellenberg, Iverson, \& McKinnon, 1999). However, as noted by the authors, performance was probably inflated because of the closed-set recognition task: Participants had matched 20 -sec excerpts of the same five songs with their title in a pretest that directly preceded the experiment. In other studies, larger amounts of musical information have been necessary to trigger familiarity or recognition in open-set recall tasks. Using the gating paradigm, these studies presented melodies note by note, in fragments of increasing duration, and participants had to identify the song or judge its familiarity. For instrumental versions of famous songs, none of the participants was able to identify any song before the third note, and songs were identified with certainty by the sixth note (Schulkind, Posner, \& Rubin, 2003). For familiar songs played with a piano timbre (i.e., without original timbre cues), nonmusicians needed on average $4.0 \pm 0.4$ notes $(2.3 \mathrm{sec})$ to perceive a melody as familiar and another 2 notes to be sure of their judgment $(5.9 \pm 0.2$ notes; $3.1 \mathrm{sec}$; Dalla Bella, Peretz, \& Aronoff, 2003). In Experiment 1, we sought to determine the least amount of information required for the emergence of familiarity with instrumental music in a situation closer to everyday listening - notably, one in which listeners did not know which items were

B. Tillmann, barbara.tillmann@olfac.univ-lyon1.fr 
being tested (an open-set task, without selective top-down processes) and in which timbre and expressive cues were provided. We used instrumental music and not songs or instrumental renderings thereof, because memory representations benefit from associations of text and tune, which are missing in instrumental music (Steinke, Cuddy, \& Jakobson, 2001).

The gating paradigm has also been used to assess the minimum duration necessary to trigger emotional judgments with music (Peretz, Gagnon, \& Bouchard, 1998). Happy- and sad-sounding musical excerpts (played with a piano timbre) were presented in segments of increasing duration, from 250 to $3,000 \mathrm{msec}$. Emotional evaluations along the happy-sad dimension required little musical information: Participants distinguished the emotional tone of 250-msec segments, even when these were presented at the beginning of the experimental session (before the longer segments). However, the excerpts differed in tempo and mode: Happy excerpts were played mostly in fast tempi and in major modes, but sad excerpts were played in slow tempi and in minor modes. As the authors stated, participants could "infer the pace of the whole excerpt by weighing the density of events contained in these very short segments" (p. 122), without processing emotional information per se. Using a different experimental paradigm, Bigand, Vieillard, Madurell, Marozeau, and Dacquet (2005) showed that emotional responses to instrumental music were not affected by shortening the excerpts from 30 to $1 \mathrm{sec}$. Even if this suggests that refined emotional responses can be triggered by short excerpts, Bigand et al. (2005) did not address whether even shorter durations might allow one to obtain emotional judgments. We addressed this question in Experiment 2, by applying the gating paradigm to instrumental music and analyzing, for the first time, the influence of the excerpts' dynamics on the time course of emotional judgments.

In sum, current research suggests that cognitive and emotional responses to music can be triggered by short stimuli, and we further investigated this issue for cognitive processing (accessing memory, triggering familiarity) in Experiment 1 and emotional judgments in Experiment 2. Given that both experiments involved the same type of musical materials (instrumental music), the same experimental procedure (gating paradigm), and nonmusician participants, they offer the possibility of making a first comparison for the speed of cognitive and emotional processes for musical material.

Our study used the gating paradigm, initially introduced for spoken word recognition (Grosjean, 1980). To minimize top-down influences, segments were presented with increasing durations (always starting with the shortest duration). This presentation excludes priming effects based on the entire items, which would lead to selective search strategies and underestimations of the required information. It has been shown that findings are not subjected to a repetition effect (confounded with increasing duration), because the same result pattern was observed for individual presentations between participants (for words, Cotton \& Grosjean, 1984) and for random presentations (for music, Peretz, Gagnon, \& Bouchard, 1998). In our study, we started with the shortest segments but did not present the increasing durations in direct succession, in order to avoid successive stimulations of the same memory entry. We used a duration-blocked presentation, which allows disrupting the effect of response preservation, negative feedback over trials and associated response strategies of participants (Walley, Michela, \& Wood, 1995): Segments of all experimental items were presented in one experimental block, and duration increased between blocks (with items presented in different random orders).

\section{EXPERIMENT 1}

\section{Method}

Participants. Fourteen students from the Université de Bourgogne participated; 13 had never taken music lessons or learned to play an instrument, and 1 had taken 7 years of private piano lessons, ending 5 years earlier.

Materials. Forty-eight excerpts from commercial CD recordings (24 familiar, 24 unfamiliar; average duration: $6.5 \pm 0.89 \mathrm{sec}$; from Plailly, Tillmann, \& Royet, 2007) of instrumental music were used. These excerpts covered a wide range of instrumentation (e.g., piano, violin, chamber music, orchestra) and composers (e.g., Bach, Johann Strauss, Vivaldi). In a posttest, 15 nonmusicians (psychology students without formal musical training) classified the excerpts as "low dynamics" or "high dynamics," depending on what they felt was the level of energy of the piece. At least $75 \%$ of the participants judged 20 excerpts to be of low dynamics and judged 24 to be of high dynamics, whereas 4 excerpts did not reach this criterion of interparticipant agreement and were considered to be ambiguous (i.e., they were excluded from the second analysis reported below). The stimuli were captured with Peak software at CD quality, and the experiment was run on PsyScope software.

Procedure. The 48 excerpts were presented in six successive blocks in segments of increasing duration $(250,500,1,000,2,000$, $4,000 \mathrm{msec}$, and complete excerpts). Participants judged the familiarity of each segment on a 10-point subjective rating scale (from $1=$ unfamiliar to $10=$ familiar). Participants were encouraged to answer even when they were unsure-notably, for 250-msec segments. Within each block, the 48 excerpts were presented in a different random order for each participant.

\section{Results}

For each participant, ratings on the complete excerpts (excluded from the ANOVAs) were used to classify items as being familiar or unfamiliar (based on a median split), and this classification was transferred to the ratings of the shorter segments: Items rated below the median were labeled "unfamiliar," and items rated above the median "familiar." Items with a rating equal to the median were eliminated from the analysis (2\%). Interestingly, participants from the pretest of Plailly et al. (2007) and from our experiment were largely in agreement in distinguishing familiar from unfamiliar categories (94\%). Overall, participants were consistent in their judgments: After they had rated an item as familiar (above their own median), they subsequently rated it as familiar for the longer durations for $83 \%$ of the items.

Familiarity judgments were analyzed with a $2 \times 5$ ANOVA with familiarity (familiar, unfamiliar) and duration $(250,500,1,000,2,000,4,000 \mathrm{msec}$, thus excluding judgments of the entire excerpts) as within-participants factors. This ANOVA revealed significant main effects of 
familiarity $\left[F(1,13)=106.06, M S_{\mathrm{e}}=3.26, p<.0001\right]$ and duration $\left[F(4,52)=51.25, M S_{\mathrm{e}}=0.62, p<.0001\right.$; Greenhouse-Geisser $\varepsilon=.50, F(1.98,25.77), p<.001]$ and a significant interaction between familiarity and duration $\left[F(4,52)=77.79, M S_{\mathrm{e}}=0.37, p<.0001\right]$; ratings were significantly higher for familiar excerpts than for unfamiliar excerpts for 500-msec $[F(1,13)=48.75, p<$ $.0001]$ and longer $(p \mathrm{~s}<.0001)$ durations, but not for 250 msec durations $(p=.13)$.

In a second analysis, the items were categorized into high- and low-dynamic excerpts, and familiarity judg- ments were analyzed with a $2 \times 5 \times 2$ ANOVA with dynamics (high, low) as an additional within-participants factor (Figure 1). This ANOVA confirmed significant main effects of familiarity $\left[F(1,13)=96.66, M S_{\mathrm{e}}=6.61\right.$, $p<.001]$ and duration $\left[F(4,52)=58.86, M S_{\mathrm{e}}=1.18\right.$, $p<.0001 ; \varepsilon=.54, F(2.15,27.95), p<.001]$ and a significant interaction $\left[F(4,52)=74.52, M S_{\mathrm{e}}=0.86, p<\right.$ $.001]$. In addition, there was a main effect of dynamics $\left[F(1,13)=207.55, M S_{\mathrm{e}}=0.24, p<.001\right]$, which was modulated by duration $\left[F(4,52)=4.64, M S_{\mathrm{e}}=0.73, p<\right.$ $.01]$ : Familiarity judgments were higher for high- than for

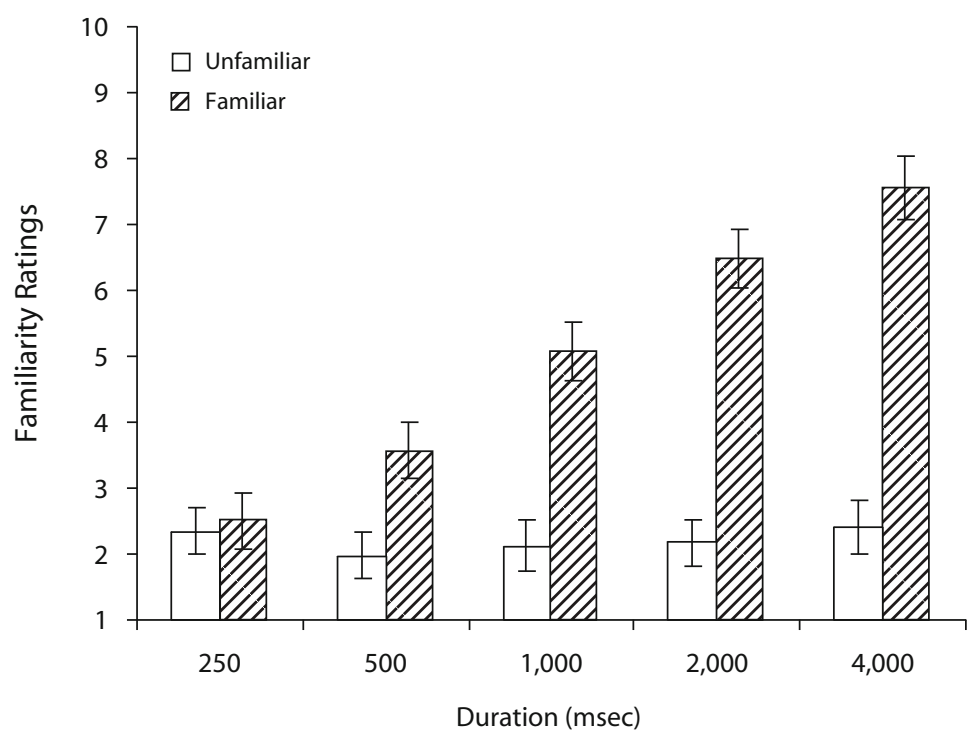

Low-Dynamic Excerpts

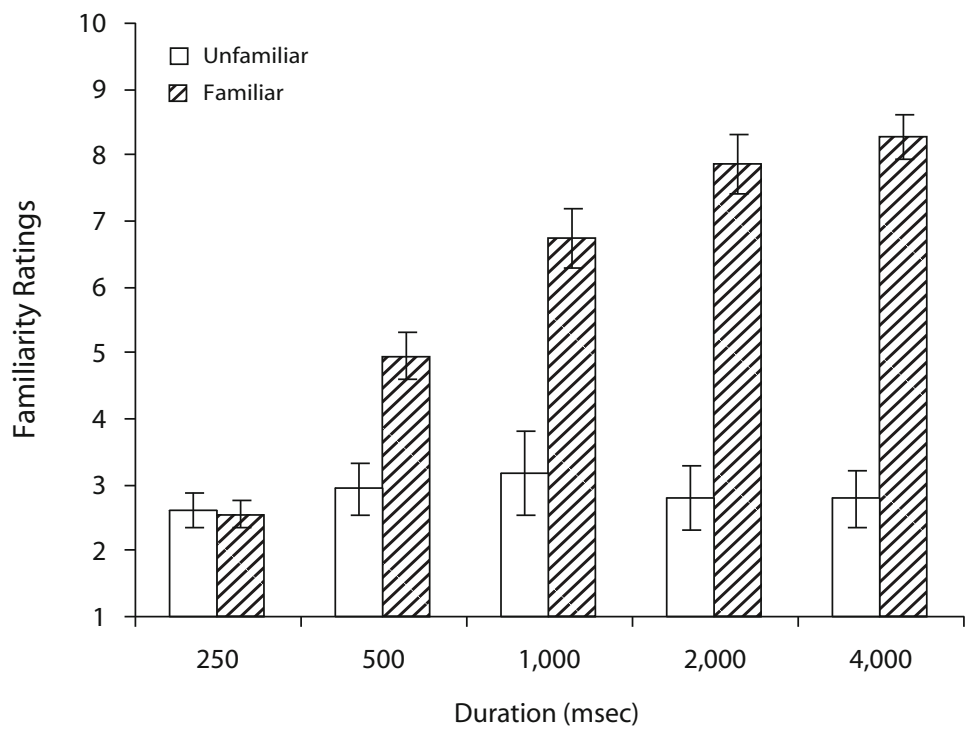

High-Dynamic Excerpts

Figure 1. Average familiarity ratings (from $1=$ unfamiliar to $10=$ familiar) in Experiment 1 for musical excerpts classified as familiar and unfamiliar (based on judgments for the complete excerpts) presented as a function of segment duration for low- (top) and high- (bottom) dynamic excerpts. Error bars represent standard errors. 
low-dynamic excerpts for all durations ( $p \mathrm{~s}<.0001)$, except for 250-msec durations ( $p=.66$ ).

\section{Discussion}

Our results show that $500 \mathrm{msec}$ of perceptual information (2.29 \pm 1.1 onsets; minimum 1, maximum 4) extracted from musical excerpts are enough to activate memory representations and to induce a feeling of familiarity. This amount of information is considerably less than the one reported by Dalla Bella et al. (2003). The difference is likely due to the use of original recordings in our study, whereas Dalla Bella et al. used melodies played with a piano timbre. Original recordings include various musical features that can trigger listeners' long-term memory of musical pieces: notes, musical structure, and dynamics, as well as timbre (instruments, spectral colors) and information linked to gestures of musical performance. Previous research has provided evidence that musical memory can store absolute attributes (e.g., pitch, timbre, tempo) of popular tunes (e.g., Halpern, 1984; Schellenberg \& Trehub, 2003).

To specify some features of the 500-msec segments that might trigger familiarity, we selected the most familiar excerpts over all participants. Most of these $500-\mathrm{msec}$ segments contained two features: a specific timbre (e.g., piano, flute, string section) and the beginning of a theme motif. For example, the first three notes (the ornamentation of the high A) and the organ timbre can evoke the Toccata in D Minor by Bach. The same note repeated three times with a specific orchestral timbre suggests the beginning of Beethoven's Fifth Symphony, even though the famous four-note opening motif is incomplete.

Experiment 1 demonstrated that a small amount of musical information is enough to trigger a cognitive process. Similarly, emotional responses to ecological stimuli are supposed to occur fast, even faster than do cognitive processes. LeDoux (1996) proposed two emotion processes: a "low road" (a "quick-and-dirty processing pathway" triggered before the stimulus has been identified) and a "high road" (a slower pathway, including cortical activity). Experiment 2 investigated the amount of musical information required for emotional judgments with the same experimental procedure as that in Experiment 1.

\section{EXPERIMENT 2}

\section{Method}

Participants. Thirty students from the Université de Bourgogne participated in Experiment 2; none had participated in Experiment 1, had taken music lessons, or had learned to play an instrument.

Materials. Twenty-eight excerpts of instrumental music (e.g., piano, chamber music, orchestra) that covered a wide range of musical periods (baroque, classical, romantic, contemporary) were used (average duration: $18.7 \pm 3.3 \mathrm{sec}$ ). They were selected by three musicologists, so that 14 excerpts were likely to be perceived as emotionally very moving and 14 excerpts were likely to be perceived as emotionally neutral, with half of the excerpts in each category being of high or low dynamics (based on tempo and instrumental density). Since finding completely neutral music was difficult, pieces of the lowest possible emotional intensity were selected as neutral. The apparatus was as described for Experiment 1.
Procedure. The 28 excerpts were presented in segments of increasing duration $(250,500,1,000,2,000,5,000 \mathrm{msec}$, and complete excerpts) in six successive blocks. Participants indicated how moving (emotionally touching, émouvant in French) the excerpts were for them on a 10-point subjective scale, from $1=$ not moving at all to $10=$ very moving. Within each block, the 28 excerpts were presented in random order for each participant.

\section{Results}

For each participant, ratings on the complete excerpts (excluded from the ANOVAs) were used to classify items as moving or neutral, and this classification was transferred to the ratings of the shorter segments: Items rated below the median were labeled "neutral," items rated above the median were labeled "moving," and items with ratings at the median were eliminated from the analysis (17\%). After participants rated an item as moving, they subsequently rated it as moving at all longer durations for $68 \%$ of the items. This consistency tended to be stronger for low- than for high-dynamic excerpts ( $72 \%$ vs. $62 \%$, $p=.095)$.

Ratings were analyzed with a $2 \times 5$ ANOVA, with emotion category (moving, neutral) and duration (250, $500,1,000,2,000$, and 5,000 msec) as within-participants factors. The main effects of emotion category $[F(1,29)=$ $\left.52.72, M S_{\mathrm{e}}=1.43, p<.0001\right]$ and of duration $[F(4,116)=$ 22. $51, M S_{\mathrm{e}}=1.43, p<.0001 ; \varepsilon=.53, F(2.14,61.97)$, $p<.001]$ were significant, as was their interaction $\left[F(4,116)=15.15, M S_{\mathrm{e}}=0.33, p<.001\right]$. The difference between moving and neutral excerpts became stronger with increasing duration but was significant even for the shortest, 250-msec duration $[F(1,29)=4.74, p<.05]$.

Items were then classified as high- and low-dynamic excerpts (see the Method section), and dynamics (high, low) was added as a within-participants factor (Figure 2). This $2 \times 5 \times 2$ ANOVA confirmed main effects of emotion $\left[F(1,26)=44.5, M S_{\mathrm{e}}=2.6, p<.001\right]$ and duration $\left[F(4,104)=20.07, M S_{\mathrm{e}}=2.59, p<.001 ; \varepsilon=.53\right.$, $F(2.12,55.2), p<.001]$ and a significant interaction $\left[F(4,104)=9.27, M S_{\mathrm{e}}=0.87, p<.001\right]$. In addition, the interaction between emotion category and dynamics $\left[F(1,26)=7.71, M S_{\mathrm{e}}=1.86, p<.05\right]$ was significant and was qualified in a three-way interaction with duration $\left[F(4,104)=3.59, M S_{\mathrm{e}}=0.45, p<.01\right]:$ Participants discriminated between moving and neutral at $250 \mathrm{msec}$ for low-dynamic excerpts $[F(1,26)=5.6, p<.05]$, but at $5 \mathrm{sec}$ for high-dynamic excerpts $[F(1,26)=25.38, p<$ $.001]$. For $250-\mathrm{msec}$ low-dynamic excerpts, the average number of tone onsets did not differ significantly between moving and neutral (1.14 vs. 1.16), and participants' judgments did not correlate significantly with number of onsets $(r=-.13)$.

A control experiment revealed that emotional judgments of 250-msec segments differed from other perceptual judgments. Fifteen new participants listened three times to the $250-\mathrm{msec}$ segments and evaluated for dull/ bright, small/tall, and sweet/salted (subjective scales from 1 to 10). For each scale, we analyzed ratings with a 2 (emotion category) $\times 2$ (dynamics) ANOVA, based on the a priori categorizations (see the Method section). For 


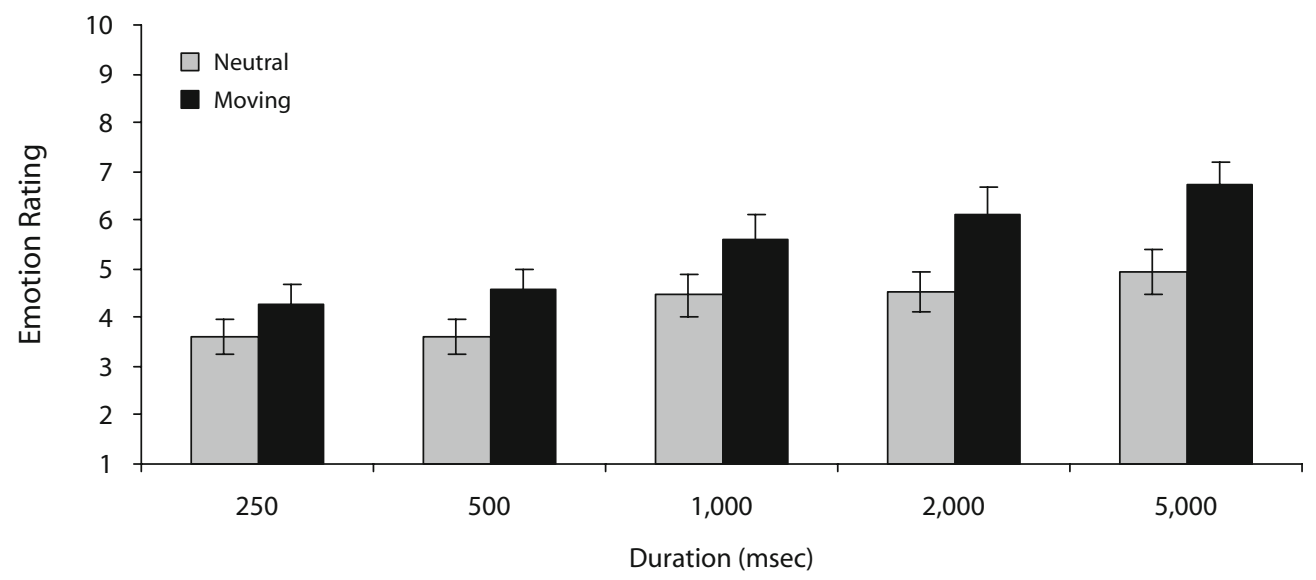

Low-Dynamic Excerpts

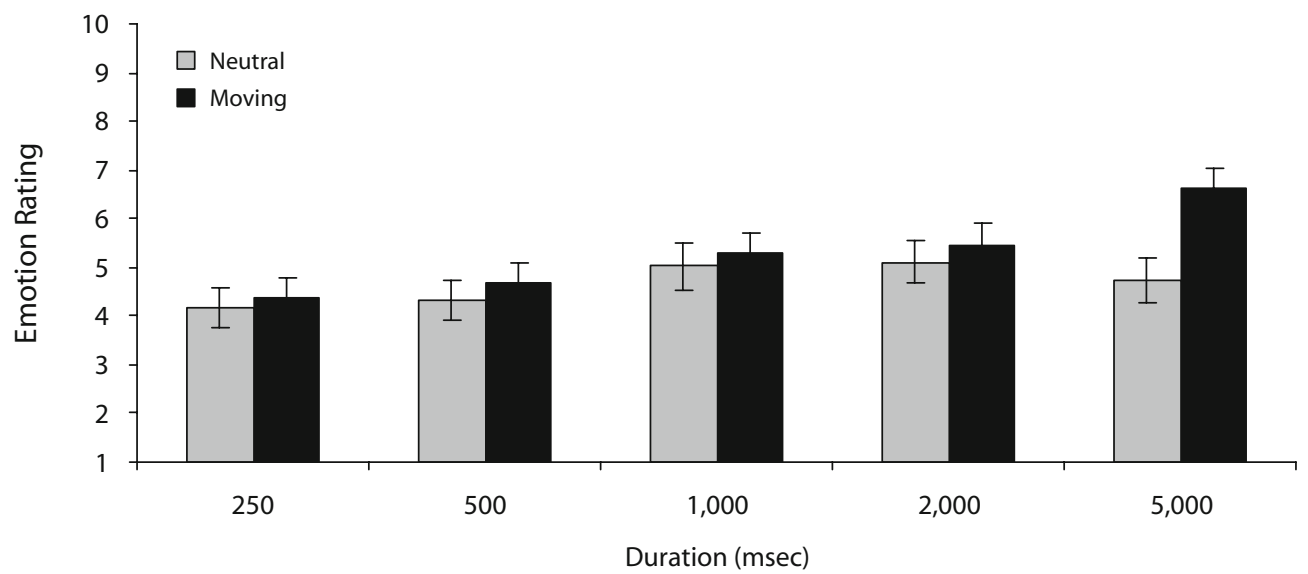

High-Dynamic Excerpts

Figure 2. Average emotion ratings (from $1=$ not moving at all to $10=$ very moving) in Experiment 2 for musical excerpts classified as neutral and moving (based on judgments for the complete excerpts) presented as a function of segment duration for low- (top) and high- (bottom) dynamic excerpts. Error bars represent standard errors.

emotional judgments, this analysis confirmed a significant interaction $[F(1,26)=11.42, p=.002]$, with moving and neutral excerpts differing at low dynamics $[F(1,26)=$ $4.99, p=.03$ ], but not at high dynamics. This pattern was not observed for the other scales. For dull/bright, main effects of emotion $[F(1,14)=29.70, p<.0001]$ and dynamics $[F(1,14)=22.42, p<.001]$, as well as their interaction $[F(1,14)=4.74, p=.047]$, were significant: Moving and neutral excerpts differed at high dynamics $[F(1,14)=17.18, p=.001]$, but not at low dynamics. The other two scales did not show interactive patterns, but only main effects of dynamics and emotion for small/tall $[F(1,14)=60.08, p<.0001 ; F(1,14)=4.62, p=.0495$, respectively] and of dynamics for sweet/salted $[F(1,14)=$ $17.06, p=.001]$.

Acoustic analyses further showed that emotional judgments were not triggered by basic acoustic feature differences in the 250-msec segments. For loudness and sensory dissonance, only the main effect of dynamics was significant - high dynamics being louder $[F(1,24)=17.21, p<$
$.001]$ and more dissonant $[F(1,24)=15.04, p<.001]$. For number of zero crossings (indicating a signal's noise level), the main effect of emotion category $[F(1,24)=4.45, p=$ $.046]$ and its interaction with dynamics $[F(1,24)=5.08$, $p=.03]$ were significant, but differences between neutral and moving excerpts were observed only for high dynamics $[F(1,24)=9.52, p=.005]$. Spectral flux (measuring quantity of spectral changes) showed a marginally significant interaction $[F(1,24)=3.35, p=.08]$, indicating differences for high dynamics only $[F(1,24)=3.39, p=$ $.08]$. For center of gravity of spectral distributions and for spectral range, no significant effects were observed.

\section{Discussion}

Experiment 2 showed that emotional judgments can be made with $250 \mathrm{msec}$ of perceptual information extracted from musical excerpts (1.26 \pm 0.53 onsets; minimum 1 , maximum 3). This finding is in agreement with Peretz, Gagnon, and Bouchard (1998), but it further reveals that this fast-acting process is not due to event density and is 
restricted to low-dynamic excerpts. At first glance, this finding seems surprising because short low-dynamic excerpts include less information (e.g., fewer notes, shorter rhythmical patterns) than do high-dynamic excerpts of the same duration. Our data suggest that information quantity is an irrelevant factor for emotional judgments. Excerpts with rapidly occurring events might be even more difficult to judge as moving, because (1) higher information quantity delays the extraction of relevant information (e.g., timbre, expression), and/or (2) they evoke a higher level of arousal, thus requiring additional time to evaluate whether the stimulating effect is produced by a moving or a neutral musical piece. In contrast, the low-dynamic excerpts might allow one to attend to the relevant information more easily, thus leading to its extraction, even with short segments.

The acoustic analyses and control experiment revealed that the features triggering emotional judgments for 250-msec segments were not basic acoustic features. This finding is in agreement with recent intracranial EEG recordings with musical stimuli, showing that emotional responses in the amygdala are not necessarily triggered by obvious acoustic features: For example, loudness changes were not systematically associated with changes in electrical activity in the amygdala, whereas more subtle changes in music (not associated with psychoacoustic properties) induced such changes (Liégeois-Chauvel et al., 2010). Both findings suggest that there are no strict correspondences between acoustic properties of the musical signal and emotional responses. Similarly to emotion perception with faces, which is based not only on single features, but also on configural and low- versus high-frequency information of the visual stimulus, emotion perception in music seems to be based on more complex feature combinations (e.g., linked to timbre and performance cues).

As was the case with previous studies using emotional judgments on facial expressions, Experiment 2 cannot differentiate whether the short excerpts induce or trigger emotion or allow for perceived emotion. Interestingly, this dissociation has become less crucial for theories of embodied cognition, suggesting that perceivers cannot provide emotional judgments without reactivating emotions internally (e.g., Niedenthal, 2007).

\section{CONCLUSION}

It has been shown previously that cognitive and emotional processes can occur rapidly for faces, voices, and words. Our study provides congruent findings with musical stimuli: A small amount of perceptual information (extracted from a musical excerpt) is sufficient for the brain to interpret this signal as music and to influence judgments of familiarity and of emotionality. Feelings of familiarity were induced after $0.5 \mathrm{sec}$, and emotional judgments were induced after $0.25 \mathrm{sec}$.

Because the gating paradigm always started with the shortest excerpts in order to minimize top-down influences, one might wonder whether ratings reflect repetition effects, particularly since mere exposure effects were also reported for music. However, it is unlikely that repetition explains our findings, because differences emerged with the first or second experimental block, and thus for first or second presentations (Experiments 2 and 1, respectively). In addition, exposure effects have been reported for unfamiliar melodies (e.g., Szpunar, Schellenberg, \& Pliner, 2004), but not for familiar melodies (Peretz, Gaudreau, \& Bonnel, 1998). This suggests that we should have observed a repetition effect for unfamiliar excerpts. In contrast, ratings of unfamiliar excerpts did not increase with increasing duration or repetition.

Our results, obtained for nonmusicians listening to instrumental classical music, demonstrated that, even if the listeners lack explicit musical expertise, a minimal amount of acoustic information is sufficient to trigger music recognition and emotional judgments. These findings show that the capacity to use little perceptual information for recognition or emotion is not restricted to faces, voices, and speech. They contribute to the ongoing debate about eventual adaptive functions of music (instead of being a purely cultural stimulus; McDermott, 2009; Peretz, 2006).

Because triggering cognitive and emotional responses to music requires only short excerpts, the independence or interaction of their processing needs to be investigated further, as has been done previously for face perception (see Posamentier \& Abdi, 2003). Our gating study investigated the smallest amount of information necessary for familiarity (accessing the musical lexicon) and emotional judgments. It provides the basis for studies that manipulate familiarity and emotional value in the same musical excerpts in order to investigate their degree of dependence (or their independence) and their time course.

\section{AUTHOR NOTE}

This research was supported by a grant from the Agence Nationale de la Recherche of the French Ministry (NT05-3_45978 "Music and Memory"). We thank Charles Delbé for help in acoustic analyses and Yannick Gérard for help in testing participants. Address correspondence concerning this article to B. Tillmann, Université Claude BernardLyon I, CNRS UMR 5020 Neurosciences Comportement Cognition, 50 Av. Tony Garnier, F-69366 Lyon Cedex 07, France (e-mail: barbara .tillmann@olfac.univ-lyon1.fr).

\section{REFERENCES}

Ashley, V., Vuilleumier, P., \& Swick, D. (2004). Time course and specificity of event-related potentials to emotional expressions. NeuroReport, 19, 211-216.

Bigand, E., Poulin, B., Tillmann, B., \& D’Adamo, D. (2003). Cognitive versus sensory components in harmonic priming effects. Journal of Experimental Psychology: Human Perception \& Performance, 29, 159-171.

Bigand, E., Vieillard, S., Madurell, F., Marozeau, J., \& DacQUET, A. (2005). Multidimensional scaling of emotional responses to music: The effect of musical expertise and of the duration of the excerpts. Cognition \& Emotion, 19, 1113-1139. doi:10.1080/ 02699930500204250

Cotton, S., \& Grosjean, F. (1984). The gating paradigm: A comparison of successive and individual presentation formats. Perception $\&$ Psychophysics, 35, 41-48.

Dalla Bella, S., Peretz, I., \& Aronoff, N. (2003). Time course of melody recognition: A gating paradigm study. Perception \& Psychophysics, 65, 1019-1028.

Eimer, M., \& Holmes, A. (2007). Event-related brain potential correlates of emotional face processing. Neuropsychologia, 45, 15-31.

Grosjean, F. (1980). Spoken word recognition processes and the gating paradigm. Perception \& Psychophysics, 28, 267-283. 
Halpern, A. R. (1984). Organization in memory for familiar songs. Journal of Experimental Psychology: Learning, Memory, \& Cognition, 10, 496-512.

Koelsch, S., Gunter, T., Friederici, A. D., \& Schröger, E. (2000). Brain indices of music processing: "Nonmusicians" are musical. Journal of Cognitive Neuroscience, 12, 520-541.

LeDoux, J. E. (1996). The emotional brain: The mysterious underpinnings of emotional life. New York: Simon \& Schuster.

liégeois-Chauvel, C., Bénar, C., Delbé, C., Bartolomei, F., Chauvel, P., \& Bigand, E. (2010). Unraveling musical emotion processing: Interaction between right auditory cortex and amygdala. Manuscript submitted for publication.

McDermotT, J. H. (2009). What can experiments reveal about the origins of music? Current Directions in Psychological Science, 18, 164-168.

Niedenthal, P. M. (2007). Embodying emotion. Science, 316, 1002 1005.

Peretz, I. (2006). The nature of music from a biological perspective. Cognition, 100, 1-32.

Peretz, I., Gagnon, L., \& Bouchard, B. (1998). Music and emotion Perceptual determinants, immediacy, and isolation after brain damage. Cognition, 68, 111-141.

Peretz, I., Gaudreau, D., \& Bonnel, A.-M. (1998). Exposure effects on music preference and recognition. Memory \& Cognition, 26, 884-902.

Plailly, J., Tillmann, B., \& Royet, J.-P. (2007). The feeling of familiarity of music and odors: The same neural signature? Cerebral Cortex, 17, 2650-2658.

Posamentier, M. T., \& Abdi, H. (2003). Processing faces and facial expressions. Neuropsychology Review, 13, 113-143.

RAPOPORT, E. (1997). Singing, mind and brain-Unit pulse, rhythm, emotion and expression. In M. Leman (Ed.), Music, gestalt, and computing: Studies in cognitive and systematic musicology (pp. 451-468). Berlin: Springer

Schellenberg, E. G., Iverson, P., \& McKinnon, M. C. (1999). Name that tune: Identifying popular recordings from brief excerpts. Psychonomic Bulletin \& Review, 6, 641-646.

SchellenberG, E. G., \& Trehub, S. E. (2003). Good pitch memory is widespread. Psychological Science, 14, 262-266.

Schulkind, M. D., Posner, R. J., \& Rubin, D. C. (2003). Musical features that facilitate melody identification: How do you know it's "your" song when they finally play it? Music Perception, 21, 217-249.

Schweinberger, S. R., Herholz, A., \& Sommer, W. (1997). Recognizing famous voices: Influence of stimulus duration and different types of retrieval cues. Journal of Speech, Language, \& Hearing Research, 40, 453-463.

SteinKe, W. R., CudDY, L. L., \& JAKoBSon, L. S. (2001). Dissociations among functional subsystems governing melody recognition after right-hemisphere damage, Cognitive Neuropsychology, 18, 411-437. doi:10.1080/02643290042000198

Szpunar, K. K., Schellenberg, E. G., \& Pliner, P. (2004). Liking and memory for musical stimuli as a function of exposure. Journal of Experimental Psychology: Learning, Memory, \& Cognition, 30, 370-381.

Tekman, H. G., \& Bharucha, J. J. (1998). Implicit knowledge versus psychoacoustic similarity in priming of chords. Journal of Experimental Psychology: Human Perception \& Performance, 24, 252260.

Walley, A. C., Michela, V. L., \& Wood, D. R. (1995). The gating paradigm: Effects of presentation format on spoken word recognition by children and adults. Perception \& Psychophysics, 57, 343-351.

WambacQ, I. J. A., Shea-Miller, K. J., \& AbubaKr, A. (2004). Nonvoluntary and voluntary processing of emotional prosody: An eventrelated potentials study. NeuroReport, 15, 555-559. doi:10.1097/ 00001756-200403010-00034

(Manuscript received March 31, 2009; revision accepted for publication December 18, 2009.) 